\title{
262 Media Reviews: Center for Organizational Learning, Innovation and Knowledge website, Institute for Innovation and Knowledge Management website, and Learning in the Modern Workplace blog
}

\author{
Review DOI 10.1108/TLO-03-2017-0028
}

In this issue, three media reviews are presented, specifically two research center websites and one blog. As already mentioned in previous issues, the general goal of this kind of reviews is to share useful information, knowledge and debates that exist on the internet, with our readers.

\section{Website review: CLIK - Center for Organizational Learning, Innovation and} Knowledge (http://wpweb2.tepper.cmu.edu/orgsci/index.html)

The CLIK (Center for Organizational Learning, Innovation and Knowledge) is a research center located at the Tepper School of Business, Carnegie Mellon University, directed by Linda Argote. Recognizing the multidisciplinary nature of learning and innovation, this center counts with researchers' contributions in several fields (organizational behavior, psychology, economics, engineering, computer science, sociology and information systems). Three research lines underlie this center's activity: behavioral foundations of group and organizational learning and innovation; social networks, learning, innovation and knowledge transfer; and technology, organizational learning and knowledge transfer. A good description of these research lines is provided on the website, including information about research topics and goals, main findings achieved, researchers involved and publications that were derived from the research work developed.

Additionally, the website also provides examples and a brief description of current research, such as "learning across product, work group and geographic boundaries", "the two wonders of experience working together: Unpacking how matching work responsibilities and individual expertise and having a common language contribute to team performance", "Mindlab project", "social networks, turnover, transactive memory systems and team performance".

A list of publications since 2005 to "in press", and conference announcements, can be also find in CLIK website.

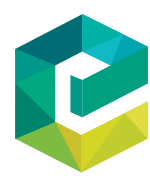

The Learning Organization Vol. 24 No. 4,2017 pp. $262-264$

(C) Emerald Publishing Limited 0969-6474

All in all, this website offers concise but useful information about the center's activity, from a description of ongoing projects to the updated list of publications. Thus, it is mostly recommended for those who are interested in topics such as transactive memory systems and performance, the effect of emotion and performance on learning, knowledge transfer across groups or learning curves.

Website: IIK - Institute for Innovation and Knowledge Management (www. esade.edu/research-webs/eng/iik)

The IIK (Institute for Innovation and Knowledge Management) is located at ESADE, Ramon Llull University, Barcelona, and is directed by Jonathan Wareham. 
Made up by a cross-disciplinary team dedicated to understanding the sources, mechanisms and consequences of innovation, the institute's "main research topics include open innovation, scientific discovery and technology transfer, platform and ecosystem design, sustainable technologies and systems of use, open government and democratizing technologies". As stated in the website, the main goal is to better understand the new systems of innovation and creativity used by organizations, "the full dynamics of their design and use, costs and benefits, creative potential and disruptive consequences".

In the research section of the website, specific themes and topics are listed around four main research areas, which are innovation and innovation management, knowledge management and organizational learning, rhetoric and narratives in management, and innovation platforms and ecosystems. These lists of specific themes and topics offer an overview of the IIK's research interests. For example, in the knowledge management and organizational learning research area, themes and topics such as the dynamics of knowledge at work, types of knowledge and evolution of knowledge in firms, democratized and "illegitimate" knowledge, networks and boundaries of organizational relations, network structure and learning performance, teams and learning groups that foster innovation and $\mathrm{R} \& \mathrm{D}$, are listed. However, a description of the research activities in each specific topic is missing, which leads the website visitor to feel there is a lack of information.

On the contrary, a good description of current and concluded projects developed by this institute can be found in the project section of the website. Here, the visitor finds a more complete vision of research activities in which IIK leads or collaborates. An example of a current project presented is the "INSPIRE: Open Innovation for SMEs" project, funded by the European Commission, which aims to professionalize open innovation processes in European small and medium-sized enterprises (SMEs), studying a critical mass of SME open innovation good practice cases and translating the findings into an integrated toolbox, a portal with resources appropriate for SMEs.

Moreover, a list of publications since 2008, as well as announcements of events can also be found on the IIK website. Of note is the section for the Center for Innovation in Cities that, under the IIK, focuses "its research on analyzing, proposing or inspiring solutions to improve the management of cities".

To sum up, this website offers useful information about the institute's activity, namely, in terms of research projects developed in European countries. It is mostly recommended for those who are interested in "where knowledge and innovation come from, how it spreads and evolves, and how it affects those who use and consume it" nowadays, in a large range of contexts, from SME's to cities.

\section{BLOG: Learning in the modern workplace (www.c4lpt.co.uk/blog/)}

This blog is about modernizing workplace learning and belongs to Jane Hart. It took the first place in the " 50 Most Socially-Shared Learning \& Development Blogs" list made by CMOE (Center for Management \& Organization Effectiveness) in 2014.

According to its focus, the visitor can find posts related to several different themes, such as gamification, social learning, training or e-learning. Moreover, as Jane Hart is the editor of Modern Workplace Learning Magazine, some posts are about articles published in the magazine. Some other posts are related to the 
newsletter that is associated with this blog. The newsletter is published weekly and provides news and articles about workplace learning, selected and commented on by Hart.

Thus, this blog is an active and updated source of information about workplace learning, where mainly practitioners can find tools, trends, knowledge and practices, as well as debate and share opinions. 